\section{MicroRNA-126 is Dysregulated in Cancer- Free Females Harboring Methylated BRCA1 Promoter through Up-Regulation of DNMTs}

\section{Abstract}

Background: Numerous microRNAs have been categorized as tumor suppressors that are often repressed by aberrant DNA methylation. Recently, BRCA1 promoter methylation in White Blood Cells (WBC) has been linked to elevated risk of developing breast cancer. In the present study, we aimed to investigative cancerrelated microRNA alterations in WBC harboring methylated BRCA1 promoter.

Methods: The Human Cancer miRNA EpiTect Methyl II Signature PCR Array was used to study the methylation status of several cancer-related microRNA sequences. Real time RT-PCR was used to determine the levels of microRNA primary transcripts and DNMTs. The Stem-loop RT-PCR assay was also used to quantify the expression levels of mature microRNAs.

Results: Out of 35 miRNAs, miR-126 was found to be hyper-methylated in WBC of breast cancer patients as well as in WBC of cancer-free females who harbored methylated $B R C A 1$ (carriers). The methylation was associated with down regulation of the pre-miR-126 in WBC and lower levels of circulating mature miR-126 in the plasma. Strong positive correlation between the expression levels of both forms of miR-126s was observed in the carriers but not in cancer patients. Importantly, we have found higher mRNA expression of DNMT1, DNMT3a and DNMT3b in WBC of carriers compared to controls.

Conclusion: Here, we have demonstrated that miR-126 is dysregulated in WBC of cancer-free females harboring methylated BRCA1 promoter. Importantly, our findings suggest that the up-regulation of DNMTs could be responsible for the aberrant methylation of both miR-126 and BRCA1 promoter in WBC.

Keywords: MicroR-126; Methylation; BRCA1; Breast cancer; DNMTs

\section{Nisreen Al-Moghrabi, Nujoud Al-Yousef, Maram Al-Showimi and Lamyaa AlGhofailli}

\author{
Department of Molecular Oncology, King \\ Faisal Specialist Hospital and Research \\ Center, KSA
}

\section{Corresponding author:}

Nisreen Al-Moghrabi

Đ nisreen@kfshrc.edu.sa

Department of Molecular Oncology, King Faisal Specialist Hospital and Research Center, Head of Cancer Epigenetic Section, KSA.

Tel: 96614647272

Citation: Al-Moghrabi N, Al-Yousef N, Al-Showimi M, et al. MicroRNA-126 is Dysregulated in Cancer-Free Females Harboring Methylated BRCA1 Promoter through Up-Regulation of DNMTs. J Clin Epigenet. 2017, 3:S1.

Received: January 16, 2017; Accepted: January 30, 2017; Published: February 06, 2017

\section{Introduction}

Epigenetics is known as inherited modifications that influence gene expression without changes in the actual DNA sequence. These modifications include DNA methylation, histone modifications and microRNA expressions. These epigenetic modifications are compelling mechanisms responsible for the inhibition of various genes, including tumor suppressors [1]. DNA methylation is one of the best-studied epigenetic modifications. There are three main DNA methyltransferases (DNMTs) responsible for establishing and maintaining the patterns of DNA methylation in human cells. DNMT3a and DNMT3b are de novo methyltransferases $[2,3]$ while DNMT1 is a maintenance enzyme [4]. The overexpression of these enzymes has been reported to correlate with poor prognosis of several cancers including breast cancer [5-9]. DNA methylation also cooperates with other epigenetic marks such as microRNAs in the complex regulation of gene expression [10].

MicroRNAs (miRs) are short non-coding RNAs that play important roles in regulating gene expression. These RNAs are a novel class of cancer-relevant molecules that are critical for proliferation, differentiation, development, apoptosis and oncogenesis [11]. Several miRs have been characterized as tumor suppressors or oncogenes [12]. The differences in the amount of circulating miRs in the blood plasma of cancer patients compared to that 
in healthy donor's offers them as innovative biomarkers for early cancer detection [13]. Several specific miRNAs play important roles in controlling DNA methylation through targeting the DNA methylation machinery. Among the reported miRNAs is miR-126. MiR-126 play crucial roles in several human cancers [14]. It acts as a metastasis suppressing microRNA that is lost in human breast cancer [15]. It has been reported that breast ductal carcinoma patients had lower circulating levels of miR126 compared to healthy controls, which correlated with that in the breast tissue [16]. In addition, microRNA-profiling study of the breast carcinoma molecular subtypes has shown that miR126 expression levels were able to differentiate malignant from benign breast tumors [17]. These data indicate that miR-126 can act as a tumor suppressor in breast cancer, which could be used as a biomarker for cancer early detection. Interestingly, the circulating levels of miR-126 were found to be significantly higher in the serum of ovarian cancer patients compared to that of controls [18].

MiR-126 is located within the $7^{\text {th }}$ intron of epidermal growth factor like domain 7 (EGFL7), which is itself one of the major targets of miR-126. Both miR-126 and EGFL7 are downregulated in cancer cell lines by hyper-methylation and their expressions can be restored with 5'-aza-2'-deoxycytidine treatment [19]. However, other studies have reported the EGFL7-independent regulation of miR-126 expression suggesting the existence of a separate promoter that derives the expression of miR-126 other than the host gene EGFL7 $[20,21]$.

Similar to germline mutations, DNA methylation is a mechanism for BRCA1 inactivation during breast cancer development [22]. The pathological features of the tumors with BRCA1 promoter methylation are similar to those carrying inherited $B R C A 1$ mutation. Both types occur at an early age and present poor histological differentiation, aneuploidy, ER and PR negativity, as well as similar global gene expression profiles $[23,24]$. The detection of methylated BRCA1 promoter in DNA from normal tissue has suggested the association of this epigenetic defect with the development of BRCA1-like breast cancer [25]. Several studies have reported the detection of methylated $B R C A 1$ in very young breast cancer patients [26] as well as in healthy females $[25,27-31]$ suggesting the potential use of methylated $B R C A 1$ as a predictor of cancer risk.

Previously, we found a clear link between the aberrant methylation of BRCA1 promoter in WBC and breast cancer-related molecular changes [32]. In the present study we show that miR126 expression is dysregulated in WBC harboring methylated $B R C A 1$ promoter through the up-regulation of DNMTs in cancerfree females.

\section{Methods}

\section{Study samples}

This is a continuation of our previous study [32]. We have made use of the available DNA and RNA isolated from WBC and plasma obtained from breast cancer patients and cancer-free females group harboring methylated $B R C A 1$ in their $\mathrm{WBC}$ and from cancer-free non-methylated females control group.

\section{Cancer miRNA DNA methylation PCR array}

The Human Cancer miRNA EpiTect Methyl II Signature PCR Array (Qiagen) was used to study promoter methylation status of a panel of 22 genes encoding 35 miRNAs. $1 \mu \mathrm{g}$ of genomic DNA isolated from WBC was used in the array following the manufacturer protocol. An integrated Excel-based template, which is provided by the manufacturer, was used for data analysis. 2.5-fold or greater change relative to controls was determined to be the threshold cut-off point for what is considered a change in gene methylation.

\section{Real-time RT-PCR}

WBC RNA was used for reverse transcription using Superscript III (Invitrogen) and random hexamer. PCR was then performed with primer specific for the S2 alternative transcript for the EGFL7 and for the DNMTs as described previously [33,34]. GAPDH mRNA was used as an internal control. Quantitative analysis was performed by real-time PCR with CYBR green using CFX96 realtime system (Bio-Rad). The fold change of mRNA expression in breast cancer patients and in carriers relative to the expression in normal controls were calculated based on the threshold cycle $(\mathrm{Ct})$ value using the $2-\Delta \Delta$ ct method.

\section{Stem-loop RT-PCR assay}

Mature miR-126 expression was analyzed in plasma. cDNA was synthesized using total RNA, gene-specific stem loop Reverse Transcription primer, and the TaqMan microRNA reversetranscription kit (Applied Biosystems). Quantitative PCR was carried out in a CFX96 real-time system (Bio-Rad) using SsoFast Supermix PCR master mix (Bio-Rad). The fold change of miRNA expression in breast cancer patients and in carriers relative to the expression in normal controls were calculated based on the threshold cycle $(\mathrm{Ct})$ value using the $2-\Delta \Delta \mathrm{ct}$ method. Experiments were normalized to small nuclear RNA U6, which was used as internal control.

\section{Statistical analysis}

Data are presented as the mean \pm SEM of replicate experiments. T-test was performed to determine the statistical significance between the different groups for gene expression levels (Patients vs. controls, Carriers vs. controls and Carriers vs. patients). All observed differences were considered to be significant when associated with a $\mathrm{P}$ value $<0.05$.

\section{Results}

\section{MiR-126is aberrantly hyper-methylated in WBC harboring methylated BRCA1promoter in both breast cancer patients and carriers}

In our previous study [20], we reported the methylated BRCA1 promoter in the WBC of 22 breast cancer patients and 13 cancerfree females by screening 155 breast cancer patients and 143 controls, respectively. For simplicity, we termed cancer-free females harboring methylated BRCA1 as "Carriers". In the present study, we have analyzed the methylation status of cancerrelated miRNAs, which are known to play important roles in 
carcinogenesis process. To this end, we used The Human Cancer miRNA EpiTect Methyl II Signature PCR Array, which profiles the promoter methylation status of a panel of 22 genes encoding 35 miRNAs. Based on a cut-off value of +2.5 fold relative to controls, miR-126 was found to be highly methylated in both breast cancer patients and carriers, 4.1 and 3.4 fold, respectively (Table 1). This indicates that WBC harboring methylated BRCA1 promoter exhibit similar alterations in microRNA expression levels in both breast cancer patients and cancer-free females.

\section{Pre-miR-126 is down-regulated in WBC of both breast cancer patients and carriers}

To investigate the effect of the aberrant hyper-methylation of miR126 DNA on the expression levels of pre-miR-126, we analyzed the level of the $\mathrm{S} 2$ alternative transcript of the EGFL7 host gene, which corresponds to the precursor of miR-126, in WBC by RealTime RT-PCR [32]. We have found that the expression of premiR-126 was not detected in the majority of the breast cancer patients resulting in significant down regulation in WBC of cancer patients compared to that of controls $(p=0.0004)$. Importantly, we have also found significant down regulation of pre-miR-126 in WBC of carriers compared to controls $(p=0.003)$ (Figure 1; Table 1). This indicates that the aberrant hyper-methylation of miR-126 in WBC associates with a down regulation of premiR-126 in both breast cancer patients and carriers.

\section{Circulating mature miR-126 is under expressed in plasma from both breast cancer patients and carriers}

It has been previously shown that miR-126 levels are significantly lower in the sera of breast cancer patients compared with those in normal controls, which correlated with that in tumor tissues [16]. Hence, we sought to investigate whether the decrease in pre-miR-126 in WBC associates with an under expressed mature miR-126 in the plasma. To this end, we made use of the TaqMan miRNA assay with gene-specific stem loop Reverse Transcription primer using total RNA isolated from plasma. We had plasma samples only from four healthy controls, four cancer patients and five carriers available for analysis. Indeed, we have found 7.6 fold decreases in the expression of circulating miR-126 in two of the patients compared to controls (Figure 2A, Table 2); while in the other two patients the expression levels were reduced 2 and 3 folds. Similarly, circulating miR-126 was decreased 2 and 3 fold in plasma from two carriers, CR5 and CR11, respectively, as compared to controls (Figure 2B, Table 3). Importantly, carrier CR21, which have a breast cancer family history (Table 3), showed a significant down regulation in the expression level of circulating miR-126, 16 fold, as compared to controls. Intriguingly, one of the

Table 1 Fold changes in methylation and mRNA expression levels in breast cancer cases and carriers.

\begin{tabular}{|l|c|c|} 
Groups & $\begin{array}{c}\text { Fold increase in } \\
\text { miR-126 promoter } \\
\text { methylation relative to } \\
\text { controls }\end{array}$ & $\begin{array}{c}\text { Fold decrease in } \\
\text { Pre-miR-126 mRNA } \\
\text { expression inside WBC } \\
\text { relative to controls }\end{array}$ \\
\hline Patients & 4.1 & $9(p=0.0004)$ \\
\hline Carriers & 3.4 & $3(p=0.003)$ \\
\hline
\end{tabular}
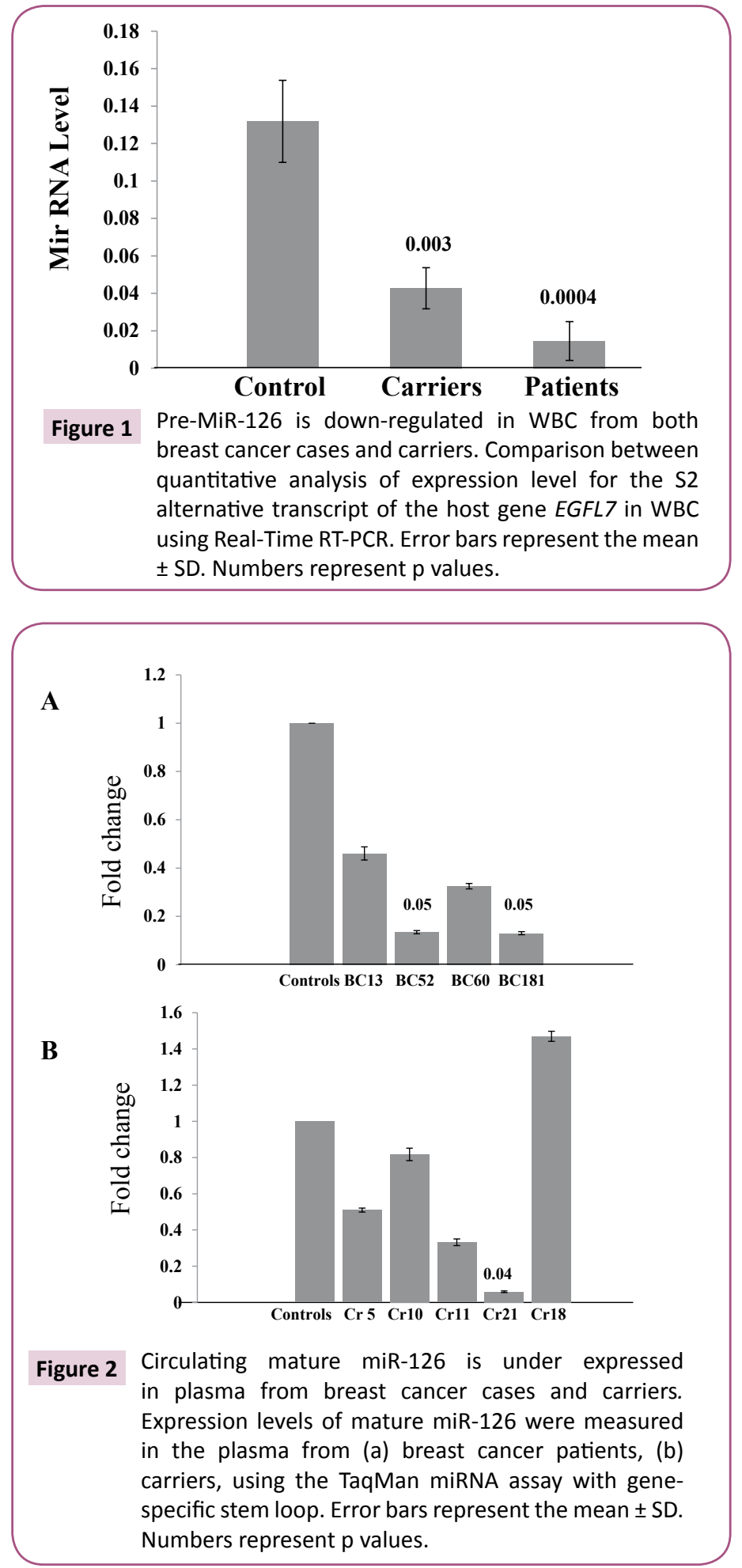

carriers, CR18 with an ovarian cancer family history (Table 3), had 1.6 fold higher levels of circulating miR-126 compared to controls (Figure 2B; Table 3). This indicates that cancer-free females with WBC methylated BRCA1 have dysregulated circulating miR-126 similar to that seen in plasma of breast cancer patients.

\section{Mir-126 is regulated at different levels in breast cancer patients and carriers}

In order to elucidate the mechanism of miR-126 biogenesis 
Table 2 Fold change in the expression of circulating miR-126 in the breast cancer patients.

\begin{tabular}{|c|c|c|}
\hline Patient samples & Fold change & $\begin{array}{c}\text { Breast cancer } \\
\text { tumor type }\end{array}$ \\
\hline BC 13 & 0.5 & ILC \\
\hline BC 52 & 0.13 & ILC \\
\hline BC 60 & 0.32 & IDC \\
\hline BC 181 & 0.13 & IDC \\
\hline
\end{tabular}

Table 3 Fold change in the expression of circulating miR-126 in the carriers.

\begin{tabular}{|c|c|c|c|}
$\begin{array}{c}\text { Carriers } \\
\text { samples }\end{array}$ & Fold change & Age & Cancer family history \\
\hline CR 5 & 0.5 & 28 & ND \\
\hline CR 10 & 0.8 & 27 & No cancer in the family \\
\hline CR 11 & 0.33 & 26 & ND \\
\hline CR 21 & 0.06 & 21 & \begin{tabular}{c} 
Breast cancer (Mother) \\
\hline CR 18
\end{tabular} \\
\hline
\end{tabular}

ND: No Data

in breast cancer patients and carriers, we sought to assess the relationship between the expression levels of the precursor and mature miR-126 in the two groups. A strong positive correlation was found between the expression levels of the two forms of miR-126 in the carriers with a correlation coefficient of 0.9 (Figure 3A). Interestingly, this was not the case in the breast cancer patients where the mature miR-126 was expressed in the plasma although the pre-miR-126 was not detected in the WBC; hence no correlation was found between the precursor and mature miR-126 (Figure 3B). This indicates that various levels of regulation govern the dysregulation of miR-126 in breast cancer patients and carriers.

\section{DNMT1, DNMT3a and DNMT3b are up-regulated in WBC of carriers}

A recent work has shown that the aberrant up-regulation of DNMT1 in esophageal squamous cell carcinomas (ESCC) is responsible for the hyper-methylation of EGFL7, which consequently leads to the down-regulation of miR-126 [34]. Moreover, it has been also reported that the overexpression of DNMT1/3a was correlated with the promoter hyper-methylation and reduced expression of BRCA1 in sporadic breast cancers [9]. Hence, it was tempting to assess the expression levels of all three DNMTs in WBC from the carriers. We used Real-Time RT- PCR utilizing primers for DNMT1, DNMT3a and DNMT3b. Intriguingly, we have found 1.7 fold $(p=0.04)$ increase in the mRNA expression level of DNMT1 (Figure 4A) and 2-fold increase for both DNMT3a $(p=0.08)$ and DNMT3b $(p=0.1)$ (Figure 4B and 4C) in carriers compared to controls. This suggests that the up-regulation of the DNMTs could be responsible for the aberrant methylation of miR126 as well as BRCA1 in WBC of carriers.

\section{Discussion}

The abnormal expression levels of mature and precursor miRNA transcripts are considered to dysregulate miRNA expression that plays an essential role in cancer initiation and progression. In the

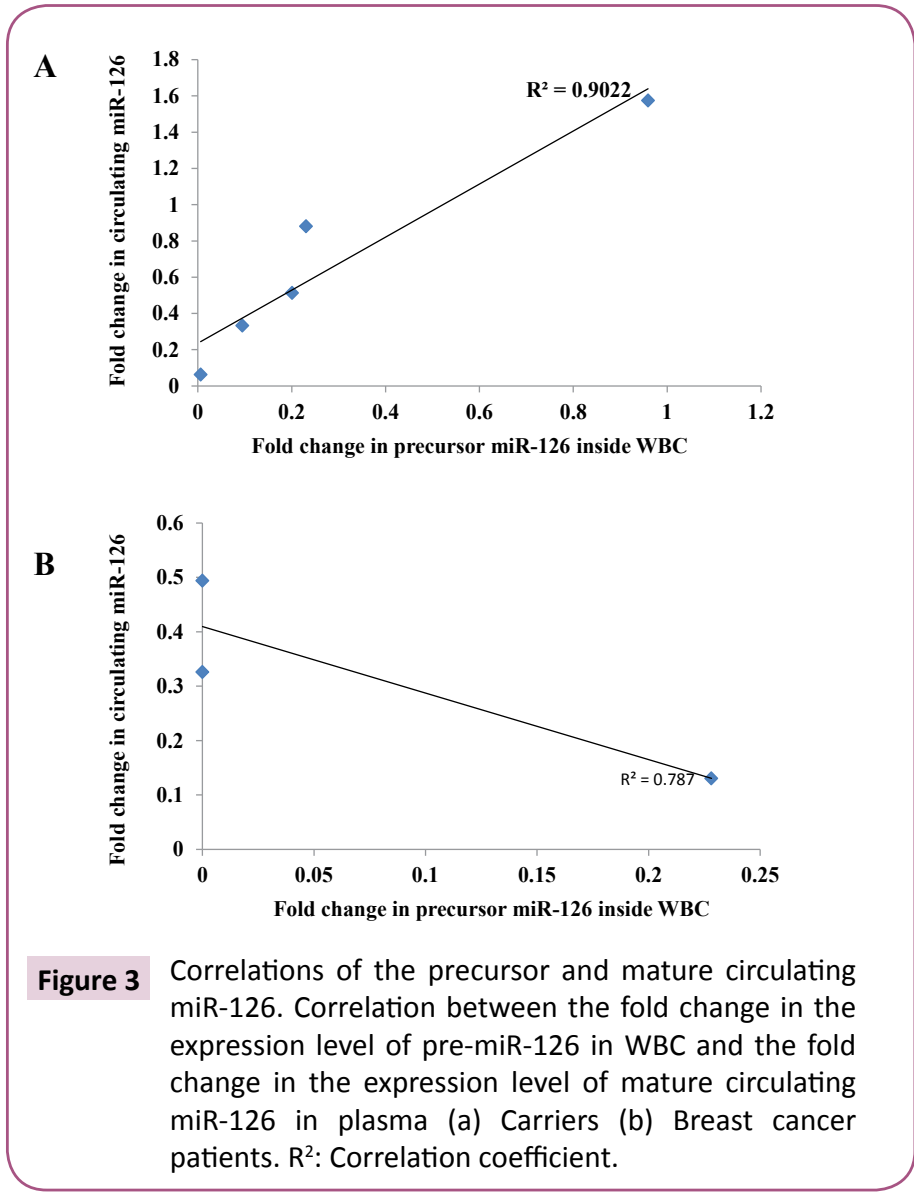

present study, we report the aberrant expression of the precursor and the mature miR-126 in WBC and plasma, respectively, in both breast cancer patients and carriers.

MicroRNA-126 has been suggested to serve as a non-invasive biomarker for breast cancer. Patients with breast cancer had lower circulating levels of miR-126 when compared to normal controls [17]. In addition, it has been shown that the down-regulation of miR-126 [35] is associated with triple-negative breast cancers. Here, we have found that miR-126 is highly methylated in WBC of both breast cancer patients and carriers. We also found that this methylation to be associated with significant decrease in premiR-126 expression in WBC from both groups.

Previously [32] we have found significant association between the presence of $B R C A 1$ promoter methylation in WBC and the cancer incidence in the families of the carriers. Interestingly, in the present study, we have also found that the dysregulation of miR-126 is associated with the cancer family history of the carriers. The carrier CR21, which have a breast cancer family history, showed the lowest expression levels of the pre-miR-126 in WBC and circulating mature miR-126 in the plasma. Moreover, the fact that carrier CR18, with an ovarian cancer family history, showed an increase in the relative expression of circulating miR126 suggest a link between BRCA1 methylation and ovarian cancer predisposition; as circulating miR-126 has been found to be up-regulated in the serum from ovarian cancer [19].

The positive correlation between WBC pre-miR-126 and mature plasma circulating miR-126 levels in the carriers suggest that miR- 


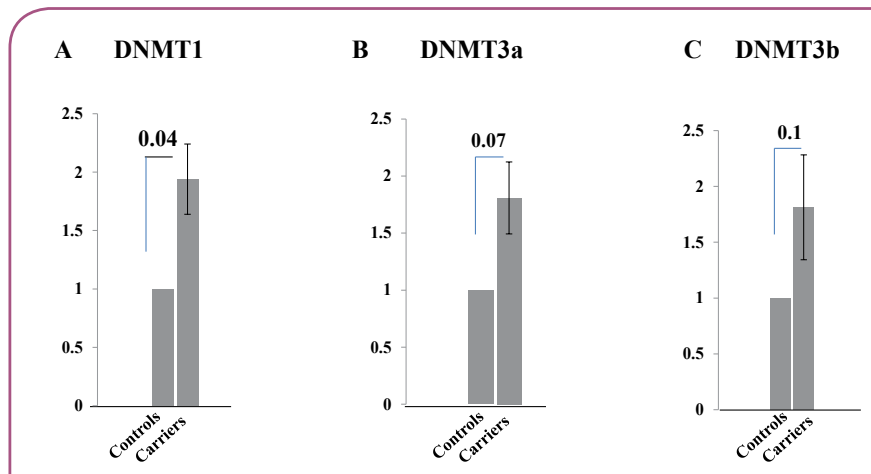

Figure 4 DNMT1, DNMT3a and DNMT3b are aberrantly upregulated in WBC from carriers. Comparison between quantitative analysis of expression levels for (a) DNMT1, (b) DNMT3a and (c) DNMT3b, in WBC using Real-Time RT- PCR. Error bars represent the mean \pm SD. Numbers represent $p$ values.

126 is regulated at the transcriptional level in these individuals. However, the lack of this correlation in the breast cancer patients also suggests that during the carcinogenesis process miR-126 is post-transcriptionally regulated. Indeed, it has been previously shown that pri-miRNA and mature expression is positively correlated in normal tissue but not in breast tumors [36]. These results suggested a multistep model for miRNA dysregulation. The fact that, in the carriers, we have found a more significant decrease in the expression level of the pre-miR-126 than the decrease in the mature form might be an indication for the multistep dysregulation of this miRNA in those individuals.

The up-regulation of DNMT1 in WBC of carriers harboring hyper-methylated miR-126 is in concordance with recent work showing the existence of a "DNMT1-miR-126 epigenetic circuit" in ESCC $[27,38]$. The aberrant up-regulation of DNMT1 in ESCC is found to be responsible for the hyper-methylation of EGFL7, which consequently leads to the down-regulation of miR126. Additionally, the up-regulation of DNMT3a and DNMT3b together with the hyper-methylation of BRCA1 in the carriers are also in agreement with the study showing a correlation of the over expression of DNMT1/3a with promoter hyper-methylation of BRCA1 in sporadic breast cancer. All in all, this may implies that the aberrant up-regulation of the DNMTs mRNA in WBC is responsible for the hyper-methylation of miR-126 as well as $B R C A 1$ in WBC from carriers.

In conclusions, we have clearly demonstrated that miR-126 is dysregulated in cancer-free women harboring methylated $B R C A 1$ promoter in their WBC. The up-regulation of the methylation machinery, DNMTs, could be responsible for the aberrant methylation of miR-126 as well as the methylation of BRCA1 promoter in WBC from carriers. All of the three DNMTs, DNMT1, DNMT3a and DNMT3b, are known to be highly up-regulated in breast tumors [39]. Our findings provide additional support for the presence of a strong link between the aberrant $B R C A 1$ promoter methylation in WBC and cancer-related molecular changes.

\section{Acknowledgment}

We are grateful to the patients and controls who participated in this stud. We would like to acknowledge Dr. Abdelilah Aboussekhra and Dr. Bedri Karakas for critically revising the article. We would like to thank the research center administration for its continuous encouragement and support. 


\section{References}

1 Jones PA, Baylin SB (2002) The fundamental role of epigenetic events in cancer. Nat Rev Genet 3: 415-428.

2 Okano M, Bell DW, Haber DA, Li E (1999) DNA methyltransferases Dnmt3a and Dnmt3b are essential for de novo methylation and mammalian development. Cell 99: 247-257.

3 Okano M, Xie S, Li E (1998) Cloning and characterization of a family of novel mammalian DNA (cytosine-5) methyltransferases. Nat Genet 19: 219-220.

4 Goll MG, Bestor TH (2005) Eukaryotic cytosine methyltransferases. Annu Rev Biochem 74: 481-514.

5 Saito Y, Kanai Y, Nakagawa T, Sakamoto M, Saito H, et al. (2003) Increased protein expression of DNA methyltransferase (DNMT) 1 is significantly correlated with the malignant potential and poor prognosis of human hepatocellular carcinomas. Int J Cancer 105 527-532.

6 Ding WJ, Fang JY, Chen XY, Peng YS (2008) The expression and clinical significance of DNA methyltransferase proteins in human gastric cancer. Dig Dis Sci 53: 2083-2089.

7 Lin RK, Hsu HS, Chang JW, Chen CY, Chen JT, et al. (2007) Alteration of DNA methyltransferases contributes to 5 ' $\mathrm{CpG}$ methylation and poor prognosis in lung cancer. Lung Cancer 55: 205-213.

8 Daniel Fl, Rivero ER, Modolo F, Lopes TG, Salum FG (2010) Immunohistochemical expression of DNA methyltransferases 1 , 3a and $3 \mathrm{~b}$ in oral leukoplakias and squamous cell carcinomas. Arch Oral Biol 55: 1024-1030.

9 Yu Z, Xiao Q, Zhao L, Ren J, Bai X, et al. (2015) DNA methyltransferase $1 / 3$ a overexpression in sporadic breast cancer is associated with reduced expression of estrogen receptor-alpha/breast cancer susceptibility gene 1 and poor prognosis. Mol Carcinog 54: 707-719.

10 Baylin SB, Jones PA (2011) A decade of exploring the cancer epigenome - biological and translational implications. Nat Rev. Cancer 11: 726-734.

11 Calin GA, Croce CM (2006) MicroRNA signatures in human cancers. Nat Rev Cancer 6: 857-866.

12 Esquela-Kerscher A, Slack FJ (2006) Oncomirs-microRNAs with a role in cancer. Nat Rev Cancer 6: 259-269.

13 Calin GA, Croce CM (2006) MicroRNA signatures in human cancers. Nat Rev Cancer 6: 857-866.

14 Mitchell PS, Parkin RK, Kroh EM, Fritz BR, Wyman SK, et al. (2008) Circulating microRNAs as stable blood-based markers for cancer detection. P Natl Acad Sci USA 105: 10513-10518.

15 Meister J, Schmidt MH (2010) miR-126 and miR-126*: new players in cancer. Scientific World J 10: 2090-2100.

16 Tavazoie SF, Alarcón C, Oskarsson T, Padua D, Wang Q, et al. (2008) Endogenous human microRNAs that suppress breast cancer metastasis. Nature 451: 147-152.

17 Wang F, Zheng Z, Guo J, Ding X (2010) Correlation and quantitation of microRNA aberrant expression in tissues and sera from patients with breast tumor. Gynecol Oncol 119: 586-593.

18 Bockmeyer CL, Christgen M, Muller M, Fischer S, Ahrens P, et al. (2011) MicroRNA profiles of healthy basal and luminal mammary epithelial cells are distinct and reflected in different breast cancer subtypes. Breast Cancer Res Treat 30: 735-745.
19 Resnick KE, Alder H, Hagan JP, Richardson DL, Croce CM, et al. (2009) The detection of differentially expressed microRNAs from the serum of ovarian cancer patients using a novel real-time PCR platform. Gynecol Oncol 112: 55-59.

20 Saito Y, Friedman JM, Chihara Y, Egger G, Chuang JC, et al. (2009) Epigenetic therapy upregulates the tumor suppressor microRNA-126 and its host gene EGFL7 in human cancer cells. Biochem Bioph Res Co 379: 726-731.

21 Monteys AM, Spengler RM, Wan J, Tecedor L, Lennox KA, et al. (2010) Structure and activity of putative intronic miRNA promoters. RNA 16: 495-505

22 Li X, Shen Y, Ichikawa H, Antes T, Goldberg GS (2009) Regulation of miRNA expression by Src and contact normalization: effects on nonanchored cell growth and migration. Oncogene 28: 4272-4283.

23 Birgisdottir V, Stefansson OA, Bodvarsdottir SK, Hilmarsdottir $\mathrm{H}$, Jonasson JG, et al. (2006) Epigenetic silencing and deletion of the BRCA1 gene in sporadic breast cancer. Breast Cancer Res 8: R38.

24 Hedenfalk I, Duggan D, Chen Y, Radmacher M, Bittner M, et al. (2001) Gene-expression profiles in hereditary breast cancer. N Engl J Med 344: 539-548.

25 Veer LJ, Dai H, van de Vijver MJ, He YD, Hart AA, et al. (2002) Gene expression profiling predicts clinical outcome of breast cancer. Nature 415: 530-536.

26 Snell C, Krypuy M, Wong EM, Kconfab I, Loughrey MB, et al. (2008) BRCA1 promoter methylation in peripheral blood DNA of mutation negative familial breast cancer patients with a BRCA1 tumour phenotype. Breast Cancer Res 10: R12.

27 Wong EM, Southey MC, Fox SB, Brown MA, Dowty JG, et al. (2011) Constitutional methylation of the BRCA1 promoter is specifically associated with BRCA1 mutation-associated pathology in early-onset breast cancer. Cancer Prev Res 4: 23-33.

28 Gupta S, Jaworska-Bieniek K, Narod SA, Lubinski J, Wojdacz TK, et al. (2014) Methylation of the BRCA1 promoter in peripheral blood DNA is associated with triple-negative and medullary breast cancer. Breast Cancer Res Treat 148: 615-622.

29 Bosviel R, Garcia S, Lavediaux G, Michard E, Dravers M, et al. (2012) BRCA1 promoter methylation in peripheral blood DNA was identified in sporadic breast cancer and controls. Cancer Epidemiol 36: e177-e182.

30 Bosviel R, Michard E, Lavediaux G, Kwiatkowski F, Bignon YJ, et al. (2011) Peripheral blood DNA methylation detected in the BRCA1 or BRCA2 promoter for sporadic ovarian cancer patients and controls. Clinica Chimica Acta 412: 1472-1475.

31 Al-Moghrabi N, Al-Qasem AJ, Aboussekhra A (2011) Methylationrelated mutations in the BRCA1 promoter in peripheral blood cells from cancer-free women. Int J Oncol 39: 129-135.

32 Iwamoto T, Yamamoto N, Taguchi T, Tamaki Y, Noguchi S (2011) BRCA1 promoter methylation in peripheral blood cells is associated with increased risk of breast cancer with BRCA1 promoter methylation. Breast Cancer Res Treat 129: 69-77.

33 Al-Moghrabi N, Nofel A, Al-Yousef N, Madkhali S, Bin Amer SM, et al. (2014) The molecular significance of methylated BRCA1 promoter in white blood cells of cancer-free females. BMC Cancer 14: 830.

34 Saito Y, Friedman JM, Chihara Y, Egger G, Chuang JC, et al. (2009) Epigenetic therapy upregulates the tumor suppressor microRNA-126 and its host gene EGFL7 in human cancer cells. Biochem Biophy. Res Commun 379: 726-731. 
35 Zheng J, Wu C, Lin Z, Guo Y, Shi L, et al. (2014) Curcumin up-regulates phosphatase and tensin homologue deleted on chromosome 10 through microRNA-mediated control of DNA methylation-a novel mechanism suppressing liver fibrosis. FEBS J 281: 88-103.

36 Liu R, Gu J, Jiang P, Zheng Y, Liu X, et al. (2015) DNMT1-microRNA126 epigenetic circuit contributes to esophageal squamous cell carcinoma growth via ADAM9-EGFR-AKT signaling. Clin Cancer Res 21: 854-863.

37 Liu Y, Cai Q, Bao PP, Su Y, Cai H, et al. (2015) Tumor tissue microRNA expression in association with triple-negative breast cancer outcomes. Breast Cancer Res Treat 152: 183-191.

38 Thomson JM, Newman M, Parker JS, Morin-Kensicki EM, Wright T, et al. (2006) Extensive post-transcriptional regulation of microRNAs and its implications for cancer. Genes Dev 20: 2202-2207.

39 Girault I, Tozlu S, Lidereau R, Bieche I (2003) Expression analysis of DNA methyltransferases $1,3 \mathrm{~A}$, and $3 \mathrm{~B}$ in sporadic breast carcinomas. Clin Cancer Res 9: 4415-4422. 